OPEN ACCESS

University of Dundee

\title{
Gefitinib and EGFR Gene Copy Number Aberrations in Esophageal Cancer
}

Petty, Russell D.; Dahle-Smith, Asa; Stevenson, David A. J.; Osborne, Aileen; Massie, Doreen; Clark, Caroline

Published in:

Journal of Clinical Oncology

DOI:

$10.1200 / J C O .2016 .70 .3934$

Publication date:

2017

Document Version

Publisher's PDF, also known as Version of record

Link to publication in Discovery Research Portal

Citation for published version (APA):

Petty, R. D., Dahle-Smith, A., Stevenson, D. A. J., Osborne, A., Massie, D., Clark, C., Murray, G. I., Dutton, S. J., Roberts, C., Chong, I. Y., Mansoor, W., Thompson, J., Harrison, M., Chatterjee, A., Falk, S. J., Elyan, S., Garcia-Alonso, A., Fyfe, D. W., Wadsley, J., ... Miedzybrodzka, Z. (2017). Gefitinib and EGFR Gene Copy Number Aberrations in Esophageal Cancer. Journal of Clinical Oncology, 35(20), 2279-2287.

https://doi.org/10.1200/JCO.2016.70.3934

\section{General rights}

Copyright and moral rights for the publications made accessible in Discovery Research Portal are retained by the authors and/or other copyright owners and it is a condition of accessing publications that users recognise and abide by the legal requirements associated with these rights.

- Users may download and print one copy of any publication from Discovery Research Portal for the purpose of private study or research.

- You may not further distribute the material or use it for any profit-making activity or commercial gain.

- You may freely distribute the URL identifying the publication in the public portal.

Take down policy

If you believe that this document breaches copyright please contact us providing details, and we will remove access to the work immediately and investigate your claim. 
Author affiliations and support information (if applicable) appear at the end of this article.

Published at jco.org on May 24, 2017.

D.R.F and Z.M. contributed equally to this work.

Clinical trial information: ISRCTN32435732, ISRCTN29580179.

Corresponding author: Russell D. Petty, $\mathrm{MBChB}, \mathrm{PhD}$, Division of Cancer

Research, School of Medicine, University of Dundee Mailbox 4, Level 7 Ninewells Hospital \& Medical School, Dundee DD 9SY Scotland, United Kingdom; e-mail: r.petty@dundee.ac.uk.

(C) 2017 by American Society of Clinical Oncology

$0732-183 X / 17 / 3520 w-2279 w / \$ 20.00$

\section{Gefitinib and EGFR Gene Copy Number Aberrations in Esophageal Cancer}

Russell D. Petty, Asa Dahle-Smith, David A.J. Stevenson, Aileen Osborne, Doreen Massie, Caroline Clark, Graeme I. Murray, Susan J. Dutton, Corran Roberts, Irene Y. Chong, Wasat Mansoor, Joyce Thompson, Mark Harrison, Anirban Chatterjee, Stephen J. Falk, Sean Elyan, Angel Garcia-Alonso, David Walter Fyfe, Jonathan Wadsley, Ian Chau, David R. Ferry, and Zosia Miedzybrodzka

\section{$\begin{array}{llllllll}\text { A } & \text { B } & \mathbf{S} & \mathbf{T} & \mathbf{R} & \mathbf{A} & \mathbf{C} & \mathbf{T}\end{array}$}

\section{Purpose}

The Cancer Esophagus Gefitinib trial demonstrated improved progression-free survival with the epidermal growth factor receptor (EGFR) tyrosine kinase inhibitor gefitinib relative to placebo in patients with advanced esophageal cancer who had disease progression after chemotherapy. Rapid and durable responses were observed in a minority of patients. We hypothesized that genetic aberration of the EGFR pathway would identify patients benefitting from gefitinib.

\section{Methods}

A prespecified, blinded molecular analysis of Cancer Esophagus Gefitinib trial tumors was conducted to compare efficacy of gefitinib with that of placebo according to EGFR copy number gain (CNG) and EGFR, KRAS, BRAF, and PIK3CA mutation status. EGFR CNG was determined by fluorescent in situ hybridization (FISH) using prespecified criteria and EGFR FISH-positive status was defined as high polysomy or amplification.

\section{Results}

Biomarker data were available for 340 patients. In EGFR FISH-positive tumors (20.2\%), overall survival was improved with gefitinib compared with placebo (hazard ratio [HR] for death, 0.59; 95\% $\mathrm{Cl}, 0.35$ to $1.00 ; P=.05)$. In EGFR FISH-negative tumors, there was no difference in overall survival with gefitinib compared with placebo (HR for death, $0.90 ; 95 \% \mathrm{Cl}, 0.69$ to $1.18 ; P=.46$ ). Patients with EGFR amplification (7.2\%) gained greatest benefit from gefitinib (HR for death, $0.21 ; 95 \% \mathrm{Cl}$, 0.07 to $0.64 ; P=.006)$. There was no difference in overall survival for gefitinib versus placebo for patients with EGFR, KRAS, BRAF, and PIK3CA mutations, or for any mutation versus none.

\section{Conclusion}

EGFR CNG assessed by FISH appears to identify a subgroup of patients with esophageal cancer who may benefit from gefitinib as a second-line treatment. Results of this study suggest that antiEGFR therapies should be investigated in prospective clinical trials in different settings in EGFR FISH-positive and, in particular, EGFR-amplified esophageal cancer.

\section{J Clin Oncol 35:2279-2287. (C) 2017 by American Society of Clinical Oncology}

\section{INTRODUCTION}

An estimated 455,000 individuals worldwide are diagnosed annually with esophageal cancer. ${ }^{1,2}$ In North America, Northern and Western Europe, and Oceania, the incidence of esophageal adenocarcinoma has risen in the last four decades and is now the predominant histologic subtype. ${ }^{2}$ Squamous cell carcinoma of the esophagus remains more common globally and in southeastern and central Asia. ${ }^{2}$

Five-year survival is only $19 \% .^{3}$ Most patients present with advanced disease not amenable to curative therapy. ${ }^{4}$ Systemic treatment with cytotoxic chemotherapy provides palliative benefits; however, current treatment options are of more limited effectiveness following progression after first-line therapy. ${ }^{4,5}$ Phase III randomized trials of second- or third-line treatment in gastric and/or esophagogastric junction adenocarcinomas have demonstrated benefit from apatinib, irinotecan, ramucurimab, and ramucurimab combined with paclitaxel. ${ }^{6-12}$ Some caution is needed in extrapolating results for gastroesophageal adenocarcinomas from different sites, because although molecular analysis suggests that esophagogastric junction and more-proximal esophageal adenocarcinomas are 
biologically similar, more-distal gastric adenocarcinomas appear distinct. ${ }^{13}$ There is more limited evidence supporting the use of second-line therapy in esophageal squamous cell carcinoma. 5,14

The Cancer Esophagus Gefitinib (COG) trial is the only randomized phase III study of second-line therapy specifically in chemoresistant esophageal cancer, including adenocarcinoma and squamous cell carcinoma. ${ }^{15}$ In the COG trial, 450 patients were randomly assigned to the epidermal growth factor receptor (EGFR) tyrosine kinase inhibitor (TKI) gefitinib or placebo. Progression-free survival (PFS) and patient-reported outcomes (PROs) were improved for gefitinib, reflecting the occurrence of rapid and durable responses to gefitinib in a minority subset of patients. Benefit from gefitinib occurred in adenocarcinomas and squamous cell carcinomas to an equal extent.

We hypothesized that the gefitinib-responsive subgroup of patients were a subset for whom EGFR signaling was an important driver. A variety of different EGFR signaling abnormalities have been described in esophageal cancer, including copy number gain $(\mathrm{CNG})$ of EGFR. ${ }^{16-20}$ Study results suggest that chromosomal instability is an early and frequent feature of esophageal cancer pathogenesis, and somatic copy number alterations occur frequently in esophageal adenocarcinoma and squamous cell carcinoma. ${ }^{19-24}$ Therefore, we hypothesized that EGFR signaling was a key pathogenic driver in the minority subset of esophageal cancers with EGFR CNG, and that these patients would benefit from gefitinib.

Accordingly, we investigated EGFR signaling pathway abnormalities in an adequately powered, prospectively collected cohort of tumor specimens from patients in the COG trial, with prespecified biomarker assays undertaken blind to treatment allocation and outcome, and a statistical analysis plan that was formulated before biomarker assay results were available.

\section{METHODS}

\section{Study Design and Oversight}

The COG trial (ISRCTN29580179) compared efficacy of $500 \mathrm{mg}$ of gefitinib daily with that of placebo in patients with esophageal cancer who had disease progression after chemotherapy. ${ }^{15}$ Participants were recruited from 48 centers in the United Kingdom and randomly assigned (1:1) to gefitinib or matching placebo by simple randomization with no stratification factors. The primary end point was overall survival (OS). Secondary end points were PFS, disease control rate (DCR; calculated as Response Evaluation Criteria in Solid Tumors [RECIST] version 1.1 partial response plus complete response plus stable disease at 8 weeks), and PROs. ${ }^{15}$ Formalin-fixed paraffin-embedded tumor tissues were prospectively collected for a translational substudy of the COG trial, TRANSCOG (ISRCTN32435732).

The TRANSCOG study was undertaken in accordance with the protocol and was approved by the National Research Ethics Service Committee (Reference 11/0372/AL). All handling and assays of tumor specimens were performed according to good clinical laboratory standards in diagnostically accredited (ISO15189:2012) laboratories. All molecular analysis was undertaken blind to treatment and clinical outcome data.

A reporting recommendations for tumor marker prognostic studies (REMARK) $)^{25}$ compliance checklist is provided (Data Supplement).

\section{Tumor Specimens}

Archived formalin-fixed paraffin-embedded tumor specimens were collected and processed according to a prespecified standard operating procedure. Central pathology review was performed to confirm histologic diagnosis and assess tumor cellularity. Tumor tissue sections $(4 \mu \mathrm{m})$ were prepared for EGFR fluorescent in situ hybridization (FISH). DNA was extracted using a standard dewaxing, tissue digestion, and phenol/ chloroform methodology with macrodissection to enrich for tumor in specimens with $<50 \%$ tumor cellularity.

\section{EGFR Gene Copy Number Analysis}

EGFR copy number analysis was by FISH and tumors were classified using the 6-point scale described previously. ${ }^{26}$ Tumors scoring 5 (high polysomy) or 6 (amplification) were classified as having EGFR high CNG and defined as EGFR FISH positive; tumors scoring 1 to 4 were classified as having no or low CNG and defined as EGFR FISH negative ${ }^{26}$ (Data Supplement). Analysis was performed by two independent scorers in a laboratory with Clinical Pathology Accreditation. Discordance led to further analysis by a third independent scorer. The testing plan and methodology were prespecified.

\section{Mutational Analysis}

Methods for each mutation were optimized for sensitivity and reliability. The final testing plan and methodology were prespecified. KRAS mutation was analyzed by pyrosequencing using primers and probes specifically designed for codons 12,13, and 61. EGFR, PIK3CA, BRAF $\mathrm{V} 600 \mathrm{E}$, and mutations were detected by Sanger sequencing as a first option; failed samples were analyzed using COBAS EGFR PIK3CA and BRAF V600E mutation testing kits (Roche Molecular Systems, Branchburg, NJ). Deletions in exon 19 of EGFR were detected by fragment length analysis. Details are available in the Data Supplement.

\section{Statistical Analysis}

The statistical analysis plan was prespecified before molecular results were available. The primary objective was to compare the effect of gefitinib with that of placebo in EGFR FISH-positive and -negative patients, and patients with and without EGFR, KRAS, BRAF, and PIK3CA mutations in the primary analysis the COG trial study population. ${ }^{15}$ The primary end point was OS; secondary end points were PFS, DCR (calculated as RECIST version 1.1 partial response plus complete response plus stable disease at 8 weeks), and PROs. We assumed that tumor samples would be available from $>300$ patients. Considering $\alpha=.05$, the accrual of tumor tissues over the 30 months of the COG study, a 12-month minimum follow-up, a hazard ratio (HR) of 0.50 favoring gefitinib in biomarker subgroups, and a median survival of 3 months in placebo-treated patients, and then assuming equal representation of gefitinib or placebo in tested samples, a predictive biomarker-defined subgroup of $10 \%$ within the gefitinib arm only comparing positive with negative biomarker groups would provide a power of $0.73,15 \%$ would provide a power of 0.88 , and $20 \%$ would provide a power of 0.93 .

The power to compare within a biomarker-positive group between gefitinib- and placebo-treated patients was reduced because of the small sample size expected in these groups (biomarker positive: $10 \%$, power of $0.45 ; 15 \%$, power of 0.61 ; and $20 \%$, power of 0.72 ). The study was not powered to test the interaction between biomarkers and treatment formally.

To estimate the treatment effects of gefitinib, we used the Cox proportional hazard model to compare outcomes in gefitinib with placebo in each biomarker-positive and -negative subgroup. The proportional hazard assumption was tested by examining the log cumulative hazards plot and Schoenfeld residual plot, and no significant deviations were found. Comparisons between biomarker status and DCR, between biomarker status and PROs, and between biomarker status and clinical variables were performed using a $\chi^{2}$ test or Fisher exact test, as appropriate. In the biomarker analysis, multiple testing was not adjusted for. To avoid errors for multiple testing in PRO analysis, biomarker status was investigated only for the four PROs of particular importance prespecified in the COG trial. ${ }^{15}$ 
The significance level for all statistical outcomes was prespecified as 0.05 and $95 \%$ CIs were calculated. The definitions of OS, PFS, and DCR, and methods for health-related quality of life (HRQL) assessment for PROs are detailed in the COG trial primary publication. ${ }^{15}$

\section{RESULTS}

\section{Patients}

Tumor specimens were available from 340 of 450 patients $(76 \%)$ in the COG study. Overall, 292 patients (65\%) had tumor evaluable for EGFR CNG by FISH and 326 patients (72\%) had tumor evaluable for EGFR, KRAS, PIK3CA, and BRAF mutation (Fig 1). The mutation analysis comprised EGFR exon 19 deletion in 254 patients, EGFR exons 18 to 21 in 223 patients, KRAS codon 12 and 13 in 268 patients, KRAS codon 61 in 287 patients, PIK3CA exon 9 in 267 patients and exon 20 in 273 patients, and $B R A F$ V600E in 267 patients.

A total of 165 patients with EGFR FISH results completed HRQL questionnaires at baseline and 4 weeks, and 88 completed them at baseline and 8 weeks; these were included in the PRO analysis (Data Supplement).

The cohorts of patients evaluable for EGFR CNG, mutations, and PROs were not different than the COG trial cohort in terms of clinical features, OS, PFS, and baseline HRQL, and clinical features were balanced in the gefitinib and placebo groups (Table 1; Data Supplement).

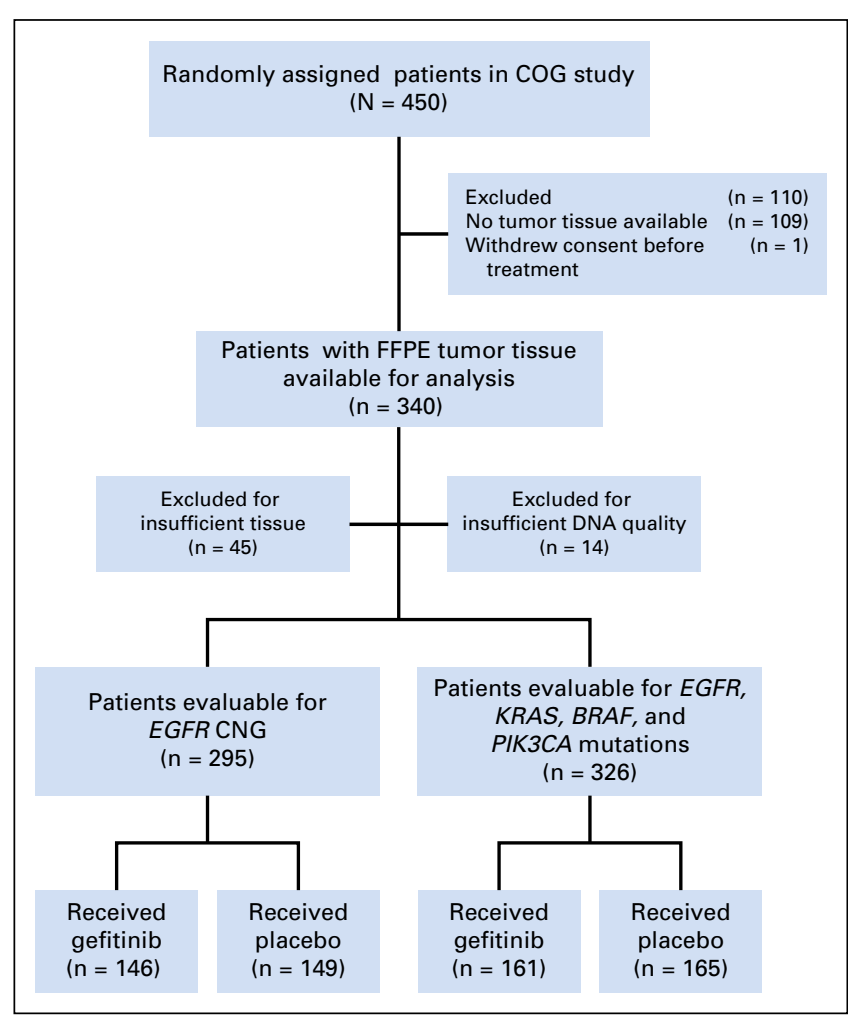

Fig 1. Patient and specimen flow in the COG and translational COG (TRANSCOG) trials. CNG, copy number gain; COG, Cancer Esophagus Gefitinib; EGFR, epidermal growth factor receptor; FFPE, formalin-fixed paraffin-embedded.
Tumors from 59 patients were EGFR FISH positive ( 59 of 292 patients; 20.2\%), with high polysomy in 13\% (38 of 292) and amplification in $7.2 \%$ (21 of 292 patients; Data Supplement). No EGFR mutations were detected. KRAS codon 12 and 13 mutations were found in $4.1 \%$ of patients ( 11 of 268), KRAS codon 61 mutations in $1.1 \%$ (three of 261), PIK3CA exon 9 mutations in $3.4 \%$ (nine of 267), PIK3CA exon 20 mutations in $0.7 \%$ (two of 273 ), and BRAF V600E in $0.4 \%$ (one of 267). There was no significant association between EGFR FISH (Table 2) or any mutation (Data Supplement) and clinical features.

\section{Efficacy According to Tumor EGFR Gene Copy Number Status}

The DCR was higher in patients with EGFR FISH-positive tumors who received gefitinib compared with those who received placebo: $37 \%$ ( 11 of 30 patients; 11 patients with stable disease) for gefitinib versus $14 \%$ (four of 29 for placebo; $P=.04$ ). PFS and OS were also improved in EGFR FISH-positive patients who received gefitinib compared with those who received placebo (PFS HR, 0.55 [95\% CI, 0.32 to 0.95 ], $P=.03$ for gefitinib $v$ placebo-treated patients; and OS HR, 0.59 [95\% CI, 0.35 to 1.00 ], $P=.05$ for gefitinib $v$ placebo-treated patients; Fig 2). OS in EGFR FISHpositive patients treated with gefitinib versus those treated with placebo at 3, 6, 9, and 12 months was $69 \%$ versus $64 \%, 38 \%$ versus $14 \%, 27 \%$ versus $5 \%$, and $13 \%$ versus $0 \%$, respectively.

A multivariate Cox proportional hazards analysis $(n=277$ for PFS and $\mathrm{n}=278$ for OS) was performed (Data Supplement) adjusted for performance status, prior treatment, body mass index, histology, disease site, age, and sex. In this analysis, PFS remained significant for benefit of gefitinib compared with placebo in EGFR FISH-positive patients (HR, $0.42 ; 95 \% \mathrm{CI}, 0.22$ to $0.81 ; P=.01$ ) but not OS (HR, $0.57 ; 95 \% \mathrm{CI}, 0.30$ to $1.06 ; P=.08$ ). None of the variables were significantly associated with PFS or OS in the multivariate analysis in EGFR FISH-positive patients.

A post hoc analysis suggested that patients with EGFR amplification (7.2\% of patients) gained greater benefit from gefitinib than those with high polysomy (Figs 2 and 3; Data Supplement).

DCR was also higher in EGFR FISH-negative patients who received gefitinib compared with those who received placebo, but DCR was greater in EGFR FISH-positive patients (25\% [29 of 115 patients receiving gefitinib], three partial responses, and 26 stable disease) versus $14 \%$ for placebo $(P=.06)$. In EGFR FISH-negative patients, PFS (HR, 0.87; 95\% CI, 0.66 to $1.12 ; P=.28$ ), and OS (HR, 0.90 ; $95 \%$ CI, 0.69 to $1.18 ; P=.46)$ were not different for gefitinib compared with that of placebo (Fig 2). OS in EGFR FISH-negative patients treated with gefitinib versus placebo at 3, 6, 9, and 12 months was $61 \%$ versus $46 \%, 33 \%$ versus $29 \%, 16 \%$ versus $22 \%$, and $8 \%$ versus $14 \%$, respectively. In the multivariate analysis, PFS and OS were not significantly different for gefitinib compared to placebo in EGFR FISH-negative patients, but performance status, prior treatment, and site of tumor were significantly associated with OS and PFS, respectively (Data Supplement).

Because of small patient numbers, differences in the COG trial-prespecified HRQL domains at 4 weeks and 8 weeks compared with baseline were not significantly different between the gefitinib and placebo groups for EGFR FISH-positive and -negative patients (Data Supplement). However in EGFR FISH-positive patients, 


\begin{tabular}{|c|c|c|c|c|}
\hline \multirow[b]{2}{*}{ Clinical Feature } & \multicolumn{2}{|c|}{ COG Trial Cohort $(\mathrm{N}=449)^{*}$} & \multicolumn{2}{|c|}{ EGFR FISH Cohort ( $N=292)$} \\
\hline & Placebo $(n=225)$ & Gefitinib ( $n=224)$ & Placebo $(n=147)$ & Gefitinib $(n=145)$ \\
\hline Age at assignment, years, mean (SD) & $64.5(9.4)$ & $63.7(9.6)$ & $64.5(9.4)$ & $64.1(9.2)$ \\
\hline \multicolumn{5}{|l|}{ Sex, No. (\%) } \\
\hline Male & $189(84.0)$ & $183(81.7)$ & $124(84.4)$ & $118(81.4)$ \\
\hline Female & $36(16.0)$ & $41(18.3)$ & $23(15.7)$ & $27(18.6)$ \\
\hline Time since diagnosis, years, median (IQR) & $0.92(0.60,1.47)$ & $0.96(0.62,1.45)$ & $0.86(0.57,1.48)$ & $0.95(0.59,1.38)$ \\
\hline \multicolumn{5}{|l|}{ Original diagnosis, No. (\%) } \\
\hline Adenocarcinoma & $168(74.7)$ & $173(77.2)$ & $102(69.4)$ & $112(77.2)$ \\
\hline Squamous & 56 (24.9) & $50(22.3)$ & 44 (29.9) & $32(22.1)$ \\
\hline Undifferentiated & $1(0.4)$ & $1(0.4)$ & $1(0.7)$ & $1(0.7)$ \\
\hline \multicolumn{5}{|l|}{ Disease site, No. (\%) } \\
\hline Esophageal & $181(80.4)$ & $171(76.3)$ & $120(81.6)$ & $109(75.2)$ \\
\hline Type I junctional & $21(9.3)$ & $26(11.6)$ & $12(8.2)$ & $17(11.7)$ \\
\hline Type II junctional & $23(10.2)$ & $27(12.1)$ & $15(10.2)$ & $19(13.1)$ \\
\hline \multicolumn{5}{|l|}{ Performance status, No. (\%) } \\
\hline 0 & 56 (24.9) & 57 (25.5) & $33(22.5)$ & $36(24.8)$ \\
\hline 1 & $125(55.6)$ & $117(52.2)$ & $86(58.5)$ & $75(51.7)$ \\
\hline 2 & $44(19.6)$ & $50(22.3)$ & $28(19.1)$ & $34(23.5)$ \\
\hline \multicolumn{5}{|l|}{ Previous treatments, No. (\%) } \\
\hline 0 & $1(0.4)$ & 0 & $1(0.7)$ & 0 \\
\hline 1 & $137(60.9)$ & $137(61.2)$ & 95 (64.6) & $94(64.8)$ \\
\hline 2 & 75 (33.3) & 78 (34.8) & $41(27.9)$ & $48(33.1)$ \\
\hline 3 & $12(5.3)$ & $9(4.0)$ & $10(6.8)$ & $3(2.1)$ \\
\hline BMI, kg/m², mean (SD); No. & 24.01 (4.77); 212 & 24.01 (4.94); 214 & $24.0(4.18) ; 141$ & $24.1(4.4) ; 139$ \\
\hline \multicolumn{5}{|l|}{ BMI grouped, No. (\%) } \\
\hline$<18.0$ & $24(10.7)$ & $24(10.7)$ & $16(10.9)$ & $13(9.0)$ \\
\hline $18.0-24.9$ & $117(52.0)$ & $113(50.5)$ & 77 (52.4) & $73(50.3)$ \\
\hline $25.0-29.9$ & $43(19.1)$ & $55(24.6)$ & $31(21.1)$ & $40(27.6)$ \\
\hline$\geq 30$ & $28(12.4)$ & $22(9.8)$ & $17(11.6)$ & $13(9.0)$ \\
\hline Missing & $13(5.8)$ & $10(4.5)$ & $6(4.1)$ & $6(4.1)$ \\
\hline
\end{tabular}

all prespecified HRQL domains improved with gefitinib compared with placebo, in contrast to an observed deterioration or lesser improvement seen in EGFR FISH-negative patients (Fig 4). At 8 weeks, mean scores for global quality of life $(+10.7)$ and difficulty eating $(-20.8)$ were improved beyond the 8-point difference considered to be of clinical importance with gefitinib compared with placebo in EGFR FISH-positive patients. However, none of the prespecified HRQL domains were changed $\geq 8$ in EGFR FISH-negative patients at 8 weeks (Data Supplement).

\section{Efficacy According to Tumor Mutation Status}

There was no significant difference in DCR, PFS, OS, or PROs for KRAS codon 12 and 13, KRAS codon 61, PIK3CA exon 9 or 20, or $B R A F \mathrm{~V} 600 \mathrm{E}$ mutations, or the presence of any mutation versus none (Data Supplement).

\section{DISCUSSION}

In the COG trial, 450 patients with esophageal adenocarcinoma or squamous cell carcinoma progressive after previous chemotherapy were randomly assigned to treatment with gefitinib or placebo. Improved DCR, PFS, and PROs were observed for gefitinib compared with placebo, reflecting rapid and durable benefits occurring in a minority subgroup. ${ }^{15}$ Gefitinib was well tolerated and, although objective responses were rare, when observed, they invariably occurred rapidly within 4 weeks of starting gefitinib. However, it is clear that most patients do not benefit from gefitinib. Identification of a predictive biomarker for patients who receive benefit from gefitinib would enable a more accurate selection of patients for treatments and prevent futile treatment in those patients who are unlikely to benefit.

Based on the outcome of the COG trial, we hypothesized that there was a subgroup of patients whose tumors were driven by EGFR signaling and who, accordingly, benefitted from treatment with gefitinib. This is analogous to non-small-cell lung cancer and colorectal adenocarcinoma in which EGFR mutation and KRAS mutation, respectively, have provided useful predictive biomarker tests and allowed subgroups to be defined as responsive to antiEGFR therapies. ${ }^{27,28}$ We aimed to determine if analysis of EGFR signaling pathway abnormalities in esophageal carcinoma would similarly predict benefit from gefitinib.

EGFR FISH-positive patients whose esophageal cancers had EGFR CNG defined as high polysomy or amplification by FISH, had improved DCR, PFS, OS, and PROs when treated with gefitinib compared with placebo. In contrast, EGFR FISH-negative patients had improved DCR, but this did not translate into improved PFS, OS, or PROs. This suggests that patients with EGFR FISH positive tumors have increased survival, as well as improved HRQL, with gefitinib, which is important in this clinical setting of limited life expectancy. Our post-hoc analysis suggests that the 


\begin{tabular}{|c|c|c|c|}
\hline Clinical Feature & EGFR Copy Number Gain $(N=59)$ & EGFR No Copy Number Gain ( $\mathrm{N}=233)$ & $P$ \\
\hline Age at assignment, years, mean (SD) & $63.9(8.3)$ & $64.4(9.5)$ & .85 \\
\hline \multicolumn{3}{|l|}{ Sex, No. (\%) } & \multirow[t]{3}{*}{.97} \\
\hline Male & 49 (83.1) & $193(82.8)$ & \\
\hline Female & $10(17.0)$ & $40(17.2)$ & \\
\hline Time since diagnosis, years median (IQR); No. & $0.95(0.51,1.27) ; 57$ & $0.90(0.60,1.45) ; 231$ & .10 \\
\hline \multicolumn{2}{|l|}{ Original diagnosis, No. (\%) } & & \multirow[t]{4}{*}{.69} \\
\hline Adenocarcinoma & $44(74.6)^{*}$ & $170(73.0)$ & \\
\hline Squamous & $14(23.7) \dagger$ & $62(26.6)$ & \\
\hline Undifferentiated & $1(1.7) \ddagger$ & $1(0.4)$ & \\
\hline \multicolumn{2}{|l|}{ Disease site, No. (\%) } & & \multirow[t]{4}{*}{.50} \\
\hline Esophageal & $46(78.0)$ & $183(78.5)$ & \\
\hline Type I junctional & $4(6.8)$ & $25(10.7)$ & \\
\hline Type II junctional & $9(15.3)$ & $25(10.7)$ & \\
\hline \multicolumn{2}{|l|}{ Performance status, No. (\%) } & & \multirow[t]{4}{*}{.27} \\
\hline 0 & $12(20.3)$ & $57(24.5)$ & \\
\hline 1 & $30(50.9)$ & $131(56.2)$ & \\
\hline 2 & $17(28.8)$ & 45 (19.3) & \\
\hline \multicolumn{2}{|l|}{ Previous treatments, No. (\%) } & & \multirow[t]{5}{*}{.90} \\
\hline 0 & 0 & $1(0.4)$ & \\
\hline 1 & $39(66.1)$ & $150(64.4)$ & \\
\hline 2 & $18(30.5)$ & $71(30.5)$ & \\
\hline 3 & $2(3.4)$ & $11(4.7)$ & \\
\hline $\mathrm{BMI}, \mathrm{kg} / \mathrm{m}^{2}$, mean (SD); No. & $23.7(4.5) ; 55$ & $24.1(4.6) ; 225$ & .55 \\
\hline \multicolumn{2}{|l|}{ BMI grouped, No. (\%) } & & \multirow[t]{6}{*}{.74} \\
\hline$<18.0$ & $4(6.8)$ & $25(10.7)$ & \\
\hline 18.0-24.9 & 33 (55.9) & $117(50.2)$ & \\
\hline $25.0-29.9$ & $14(23.7)$ & 57 (24.5) & \\
\hline$\geq 30$ & $4(6.8)$ & $26(11.2)$ & \\
\hline Missing & $4(6.8)$ & $8(3.4)$ & \\
\hline \multicolumn{4}{|c|}{$\begin{array}{l}\text { Abbreviations: BMI, body mass index; EGFR, epidermal growth factor receptor; IQR, interquartile range; SD, standard deviation. } \\
\text { *Twenty-nine of } 44 \text { EGFR fluorescent in situ hybridization (FISH) -positive adenocarcinomas (65.9\%) had EGFR high polysomy and } 15 \text { of } 44 \text { (34.1\%) had EGFR } \\
\text { amplification. } \\
\text { †Fourteen of } 14 \text { EGFR FISH-positive squamous cell carcinomas (64.2\%) had EGFR high polysomy and five of } 14(35.8 \%) \text { had EGFR amplification. } \\
\text { fThe EGFR FISH-positive undifferentiated carcinoma was EGFR amplified. }\end{array}$} \\
\hline
\end{tabular}

benefit of gefitinib is greater in those with EGFR-amplified tumors than with high polysomy tumors. Our study was not powered to investigate these subgroups, and additional investigation is needed to validate this observation. However, this finding is consistent with results for anti-EGFR and other targeted therapies in other tumor types. ${ }^{29-31}$ Overall, our results suggest it is likely there is a greater benefit from gefitinib in EGFR-amplified esophageal cancers compared with those with high polysomy.

Our findings are supported by the report of high sensitivity to gefitinib in a primary cell line derived from a patient with esophageal adenocarcinoma with EGFR high polysomy. ${ }^{32}$ In addition, a single-arm phase II trial of the EGFR TKI icotinib in esophageal squamous cell carcinoma with EGFR CNG determined by FISH or strongly positive EGFR immunohistochemistry reported a DCR of $46 \%$. $^{33}$

This suggests that EGFR FISH identifies those patients with esophageal cancer whose tumors are driven by EGFR signaling and for whom inhibition of EGFR confers benefit. Investigating the impact of EGFR FISH positivity on sensitivity to EGFR inhibitors other than gefitinib would test this hypothesis. Similar to HER2 in gastroesophageal adenocarcinoma, EGFR TKIs and monoclonal antibodies may have different impacts in EGFR FISH-positive patients. $^{34,35}$ Not all EGFR FISH-positive patients benefit from gefitinib and coamplification of other receptor tyrosine kinases (RTKs) and/or downstream signaling pathways may also be important determinants of clinical benefit. ${ }^{36,37}$ RTK pathway copy number profiling could improve predictive accuracy and guide personalized use of EGFR and other RTK inhibitors.

To our knowledge, there have been no previous randomized trials of second-line therapy in esophageal cancer including adenocarcinomas and squamous cell carcinomas. However, the PFS and OS benefits from gefitinib compared with placebo in EGFR FISH-positive patients that we observed is of a similar proportion to those in randomized studies versus placebo or supportive care only for other second-line therapies in gastric and gastroesophageal junction adenocarcinoma, including apatinib, regorafenib, docetaxel, irinotecan, and ramucurimab. ${ }^{7-12,38}$ In comparison with docetaxel and irinotecan, the toxicity of gefitinib is preferable. The toxicity of gefitinib is similar overall to that of ramucurimab, apatinib, or regorafenib, but because there are no predictive biomarkers for these agents, the use of gefitinib in patients selected by EGFR FISH status represents an alternative with increased clinical and cost effectiveness.

The use of next-generation sequencing would have provided higher sensitivity for subclonal mutations. However, the low frequency of mutations detected in our study, in contrast to EGFR CNG, which predicts gefitinib benefit, is consistent with other reports and genome landscaping studies that demonstrate predominant copy number changes. ${ }^{18-20,36}$ In adenocarcinoma, 


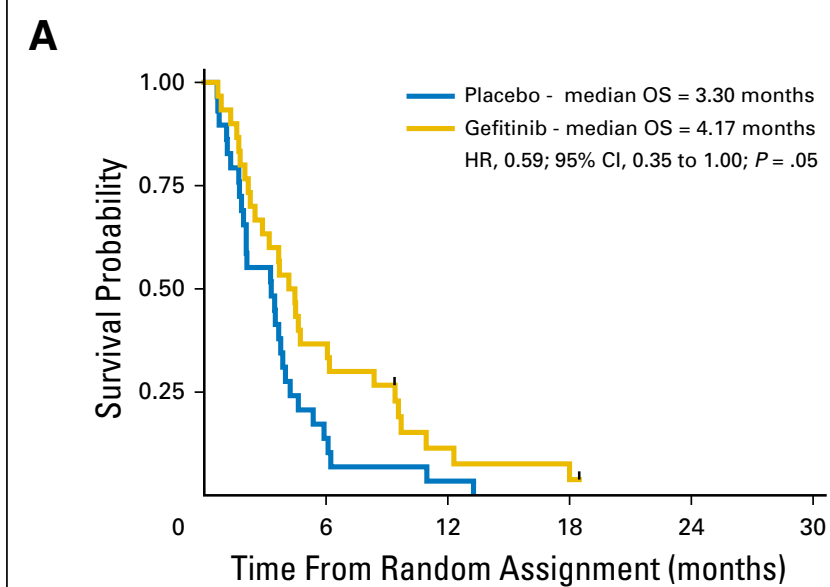

No. at risk

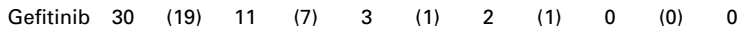

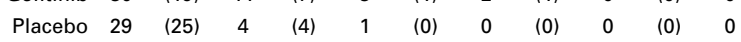

C

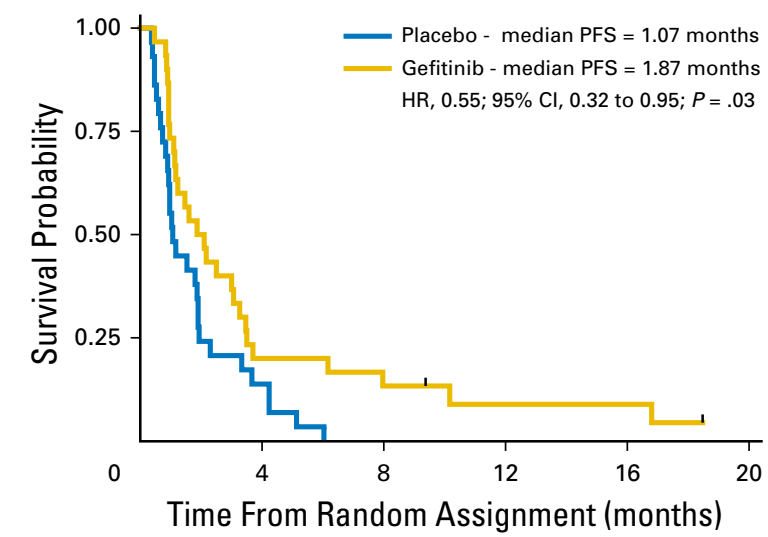

No. at risk

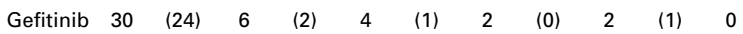

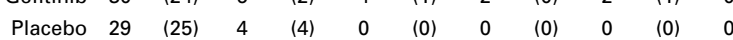

E

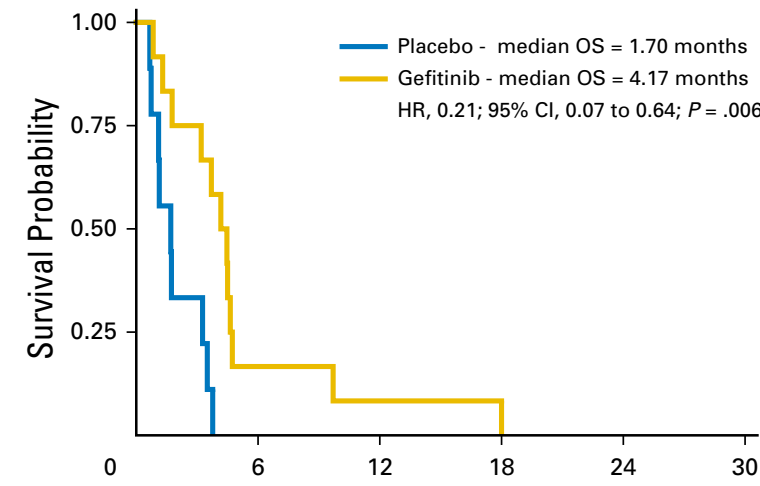

Time From Random Assignment (months)

No. at risk

$\begin{array}{cccccccccccc}\text { Gefitinib } & 12 & (10) & 2 & (2) & 1 & (0) & 1 & (0) & 0 & (0) & 0 \\ \text { Placebo } & 9 & (9) & 0 & (0) & 0 & (0) & 0 & (0) & 0 & (0) & 0\end{array}$

B

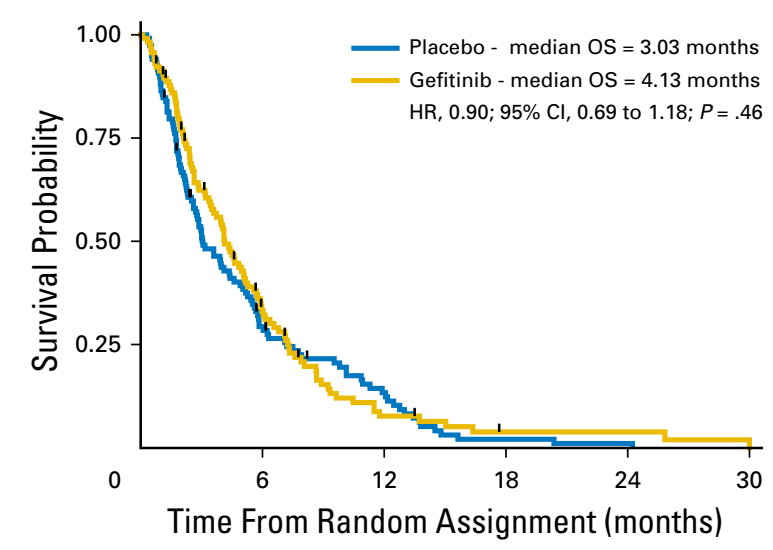

No. at risk

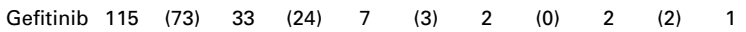

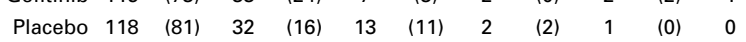

D

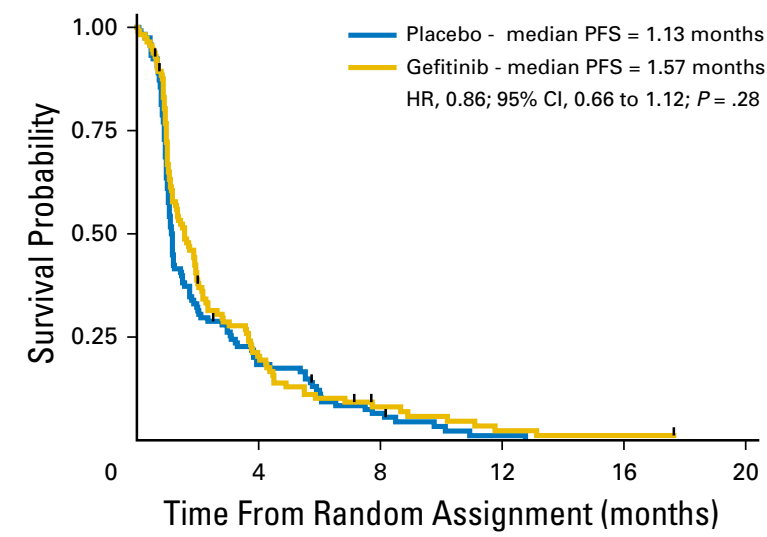
No. at risk

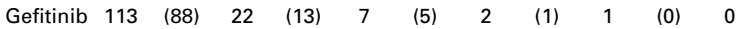

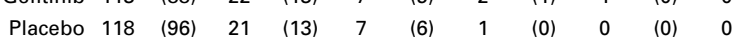

$\mathbf{F}$

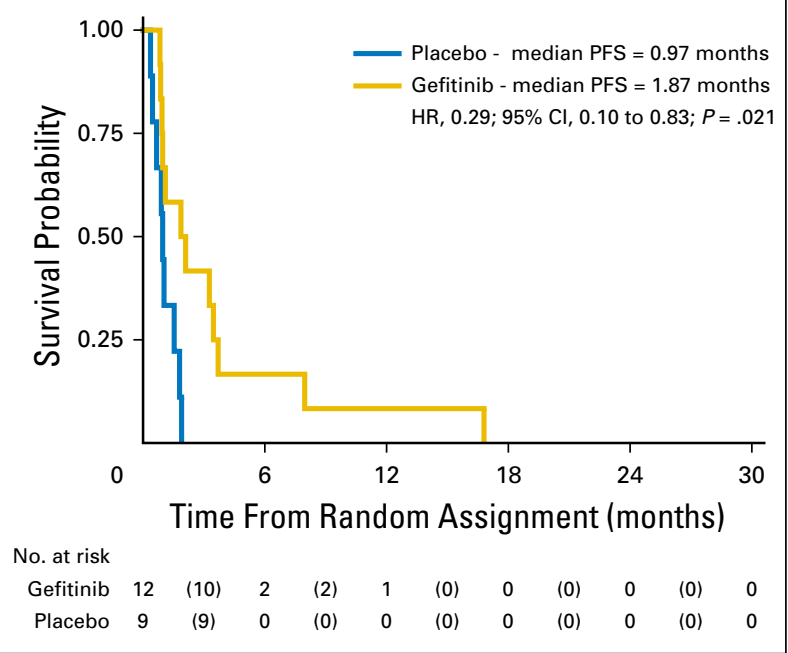

Fig 2. Kaplan-Meier estimates of PFS and OS according to treatment group. (A) OS in patients positive for EGFR by fluorescent in situ hybridization (FISH). (B) OS in EGFR

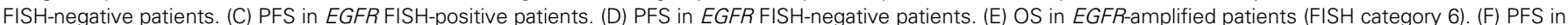
EGFR-amplified patients (FISH category 6). EGFR, epidermal growth factor receptor; HR, hazard ratio; OS, overall survival; PFS, progression-free survival. 
A

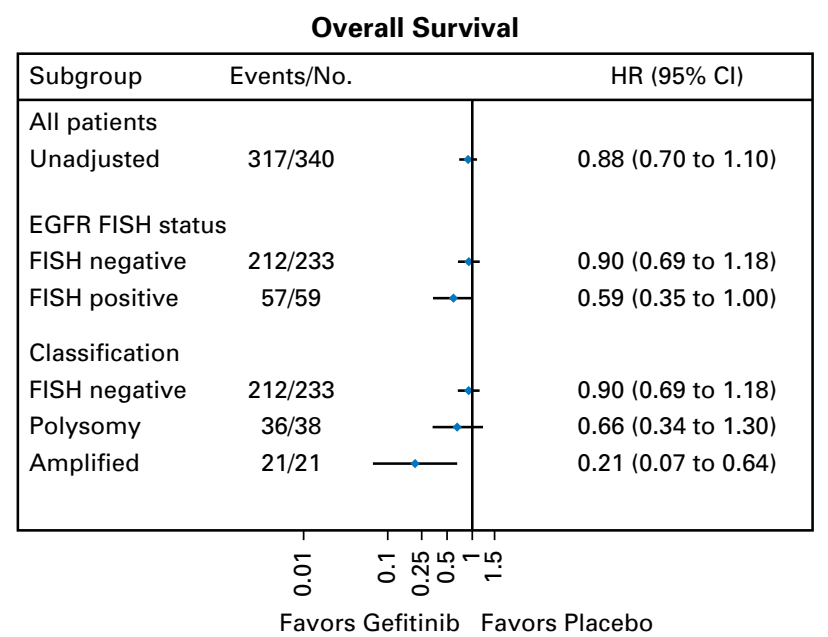

B

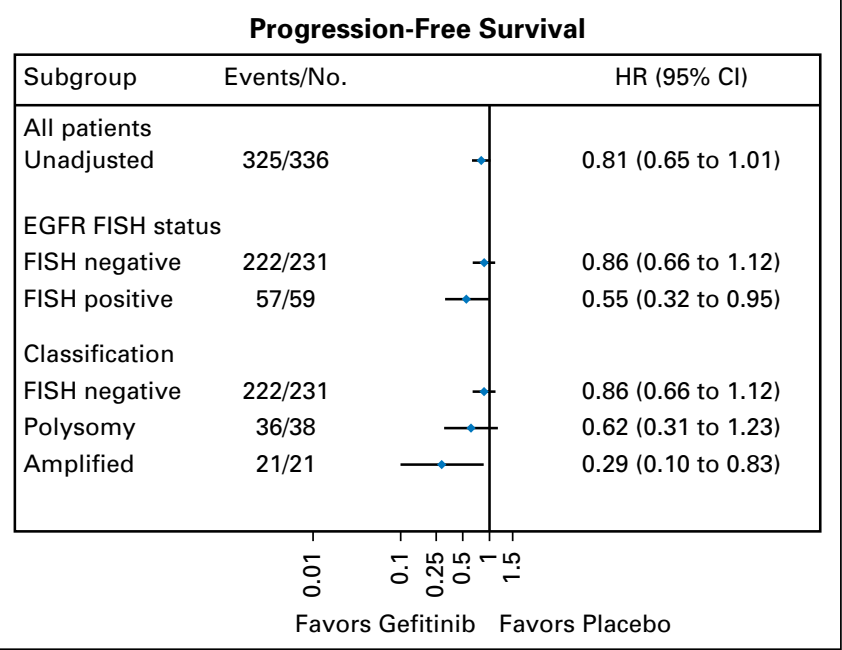

Fig 3. Forest plot for EGFR FISH-positive and -negative patients, and EGFR high polysomy and amplification. (A) OS. (B) PFS. CNG, copy number gain; EGFR, epidermal growth factor receptor; FISH, fluorescent in situ hybridization; HR, hazard ratio.

chromosomal instability leading to structural aneuploidy including CNGs of oncogenes such as EGFR is common. ${ }^{17-20,23,36}$ In our analysis, we found no significant difference in the frequency of $E G F R$ CNG between adenocarcinomas and squamous cell carcinomas.
There was insufficient tissue available to analyze EGFR protein expression. In esophageal cancer, EGFR FISH-positive tumors almost invariably overexpress EGFR by immunohistochemistry, but up to $50 \%$ of EGFR FISH-negative tumors also strongly

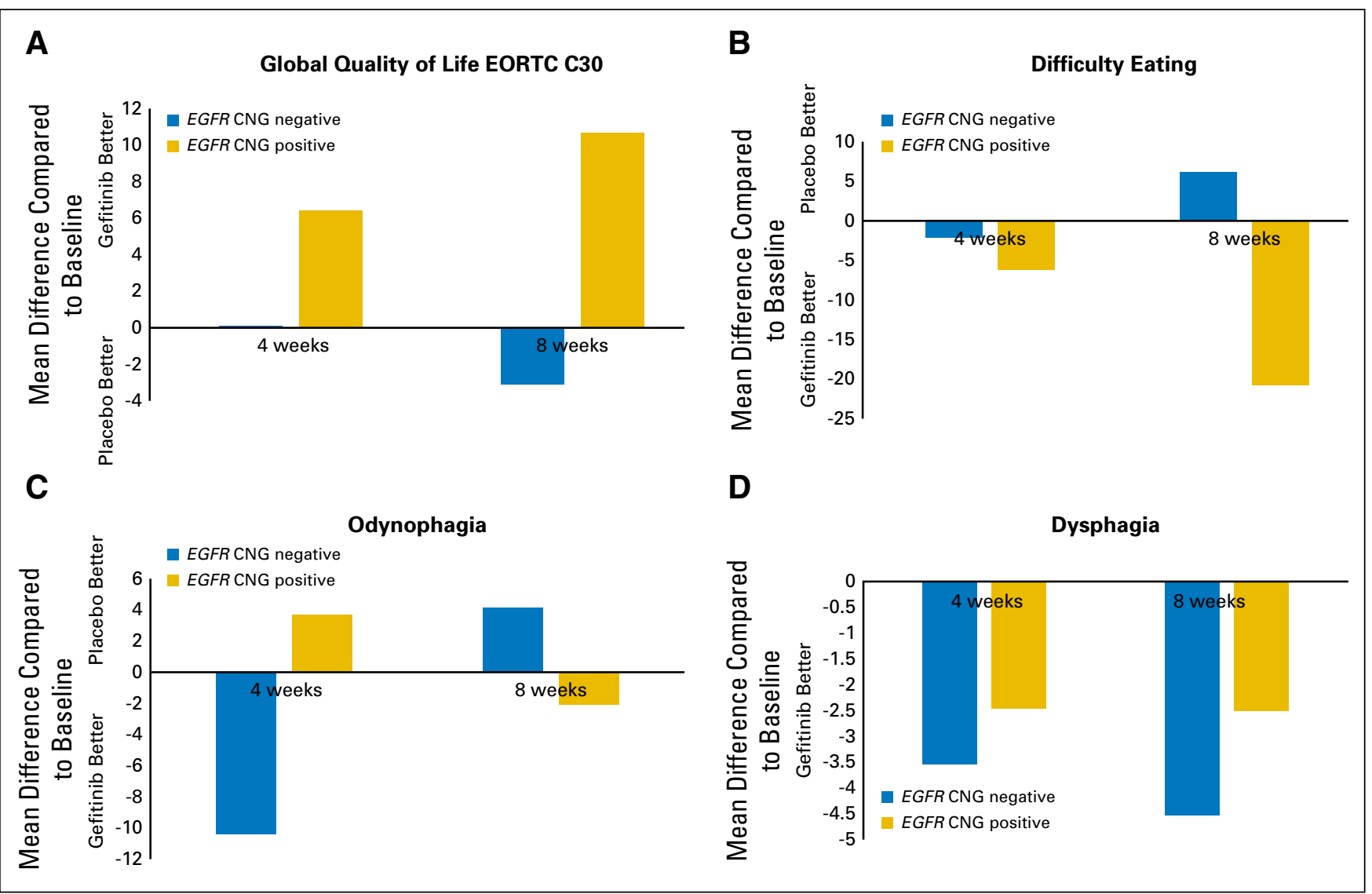

Fig 4. Patient-reported outcomes. (A) Global quality of life. (B) Difficulty eating. (C) Odynophagia. (D) Dysphagia. CNG, copy number gain; EGFR, epidermal growth factor receptor; FISH, fluorescent in situ hybridization. 
overexpress EGFR protein. ${ }^{39,40}$ Together with our demonstration of lack of benefit from gefitinib in EGFR FISH-negative patients, this suggests that EGFR FISH may be more a reliable predictive biomarker than EGFR immunohistochemistry, although this needs to be confirmed by additional investigation.

This study was retrospective and, therefore, subject to limitations. The results of EGFR CNG and the mutation analysis may not be representative of the intention-to-treat population from the original randomization. However, the cohort of patients tested did not show significant differences in clinical features compared with the intention-to-treat population. Furthermore, molecular testing and analysis were hypothesis driven, performed to diagnostic standard in a reference laboratory with clinical pathology accreditation, blind to patient treatment and outcomes, had a prospectively determined statistical analysis plan formulated before molecular results were available, and used data from a large, randomized controlled trial. Therefore, this study robustly evaluated EGFR CNG determined by FISH as a predictive biomarker.

In conclusion, EGFR FISH appears to predict a benefit from gefitinib in patients with esophageal cancer whose disease has progressed after previous chemotherapy. The role of gefitinib and other anti-EGFR therapies should be explored in prospective clinical trials in different settings in EGFR FISH-positive esophageal cancer, particularly in EGFR-amplified tumors, in which the impact of these agents is likely to be greatest.
AUTHORS' DISCLOSURES OF POTENTIAL CONFLICTS OF INTEREST

Disclosures provided by the authors are available with this article at jco.org.

\section{AUTHOR CONTRIBUTIONS}

Conception and design: Russell D. Petty, David A.J. Stevenson, Susan J. Dutton, David R. Ferry, Zosia Miedzybrodzka

Provision of study materials or patients: Russell D. Petty, Wasat Mansoor, Joyce Thompson, Mark Harrison, Anirban Chatterjee, Stephen J. Falk, Sean Elyan, Angel Garcia-Alonso, David Walter Fyfe, Jonathan Wadsley, Ian Chau, David R. Ferry, Zosia Miedzybrodzka

Collection and assembly of data: Russell D. Petty, Asa Dahle-Smith, David A.J. Stevenson, Aileen Osborne, Doreen Massie, Caroline Clark, Graeme I. Murray, Susan J. Dutton, Corran Roberts, Irene Y. Chong, Jonathan Wadsley, David R. Ferry, Zosia Miedzybrodzka

Data analysis and interpretation: Russell D. Petty, Asa Dahle-Smith, David A.J. Stevenson, Aileen Osborne, Doreen Massie, Caroline Clark, Graeme I. Murray, Susan J. Dutton, Corran Roberts, Irene Y. Chong, Wasat Mansoor, Joyce Thompson, Mark Harrison, Anirban Chatterjee, Stephen J. Falk, Sean Elyan, Angel Garcia-Alonso, David Walter Fyfe, Ian Chau, David R. Ferry, Zosia Miedzybrodzka

Manuscript writing: All authors

Final approval of manuscript: All authors

Accountable for all aspects of the work: All authors

\section{REFERENCES}

1. Torre LA, Bray F, Siegel RL, et al: Global cancer statistics, 2012. CA Cancer J Clin 65:87-108, 2015

2. Arnold M, Soerjomataram I, Ferlay J, et al: Global incidence of oesophageal cancer by histological subtype in 2012. Gut 64:381-387, 2015

3. National Cancer Institute Surveillance, Epidemiology, and End Results Program: SEER Cancer Statistics Review, 1975-2013. http://seer.cancer.gov/ csr/1975_2013

4. Pennathur A, Gibson MK, Jobe BA, et al: Oesophageal carcinoma. Lancet 381:400-412, 2013

5. Thallinger CMR, Raderer $M$, Hejna $M$ : Esophageal cancer: A critical evaluation of systemic second-line therapy. J Clin Oncol 29:4709-4714, 2011

6. Wilke $H$, Muro $K$, Van Cutsem $E$, et al: Ramucirumab plus paclitaxel versus placebo plus paclitaxel in patients with previously treated advanced gastric or gastro-oesophageal junction adenocarcinoma (RAINBOW): A double-blind, randomised phase 3 trial. Lancet Oncol 15:1224-1235, 2014

7. Fuchs CS, Tomasek J, Yong CJ, et al: Ramucirumab monotherapy for previously treated advanced gastric or gastro-oesophageal junction adenocarcinoma (REGARD): An international, randomised, multicentre, placebo-controlled, phase 3 trial. Lancet 383:31-39, 2014

8. Ford HER, Marshall $A$, Bridgewater JA, et al: Docetaxel versus active symptom control for refractory oesophagogastric adenocarcinoma (COUGAR-02): An open-label, phase 3 randomised controlled trial. Lancet Oncol 15:78-86, 2014

9. Kang JH, Lee SI, Lim DH, et al: Salvage chemotherapy for pretreated gastric cancer: A randomized phase III trial comparing chemotherapy plus best supportive care with best supportive care alone. J Clin Oncol 30:1513-1518, 2012

10. Thuss-Patience PC, Kretzschmar A, Bichev D, et al: Survival advantage for irinotecan versus best supportive care as second-line chemotherapy in gastric cancer-a randomised phase III study of the Arbeitsgemeinschaft Internistische Onkologie (AIO). Eur J Cancer 47:2306-2314, 2011

11. Janowitz $T$, Thuss-Patience $P$, Marshall $A$, et al: Chemotherapy vs supportive care alone for relapsed gastric, gastroesophageal junction, and oesophageal adenocarcinoma: A meta-analysis of patient-level data. Br J Cancer 114:381-387, 2016

12. Li J, Qin S, Xu J, et al: Randomized, doubleblind, placebo-controlled phase III trial of apatinib in patients with chemotherapy-refractory advanced or metastatic adenocarcinoma of the stomach or gastroesophageal junction. J Clin Oncol 34:1448-1454, 2016

13. Hayakawa $Y$, Sethi $N$, Sepulveda $A R$, et al: Oesophageal adenocarcinoma and gastric cancer: Should we mind the gap? Nat Rev Cancer 16: 305-318, 2016

14. Dahle-Smith A, Petty RD: Biomarkers and novel agents in esophago-gastric cancer: Are we making progress? Expert Rev Anticancer Ther 15: 1103-1119, 2015

15. Dutton SJ, Ferry DR, Blazeby JM, et al: Gefitinib for oesophageal cancer progressing after chemotherapy (COG): A phase 3, multicentre, double-blind, placebo-controlled randomised trial. Lancet Oncol 15:894-904, 2014

16. Lin DC, Hao JJ, Nagata $Y$, et al: Genomic and molecular characterization of esophageal squamous cell carcinoma. Nat Genet 46:467-473, 2014

17. Song $Y, \operatorname{Li} L, O u Y$, et al: Identification of genomic alterations in oesophageal squamous cell cancer. Nature 509:91-95, 2014
18. Dulak AM, Stojanov $P$, Peng $S$, et al: Exome and whole-genome sequencing of esophageal adenocarcinoma identifies recurrent driver events and mutational complexity. Nat Genet 45:478-486, 2013

19. Ross-Innes CS, Becq J, Warren A, et al: Whole-genome sequencing provides new insights into the clonal architecture of Barrett's esophagus and esophageal adenocarcinoma. Nat Genet 47: 1038-1046, 2015

20. Stachler MD, Taylor-Weiner A, Peng $S$, et al: Paired exome analysis of Barrett's esophagus and adenocarcinoma. Nat Genet 47:1047-1055, 2015

21. Nones K, Waddell N, Wayte N, et al: Genomic catastrophes frequently arise in esophageal adenocarcinoma and drive tumorigenesis. Nat Commun 5: 5224, 2014

22. Li $X$, Galipeau PC, Paulson TG, et al: Temporal and spatial evolution of somatic chromosomal alterations: A case-cohort study of Barrett's esophagus. Cancer Prev Res (Phila) 7:114-127, 2014

23. Murugaesu N, Wilson GA, Birkbak NJ, et al: Tracking the genomic evolution of esophageal adenocarcinoma through neoadjuvant chemotherapy. Cancer Discov 5:821-831, 2015

24. Beroukhim $\mathrm{R}$, Mermel $\mathrm{CH}$, Porter $\mathrm{D}$, et al: The landscape of somatic copy-number alteration across human cancers. Nature 463:899-905, 2010

25. McShane LM, Altman DG, Sauerbrei W, et al: Reporting recommendations for tumor marker prognostic studies. J Clin Oncol 23:9067-9072, 2005

26. Dahle-Smith $\AA$, Stevenson $D$, Massie $D$, et al: Epidermal growth factor (EGFR) copy number aberrations in esophageal and gastro-esophageal junctional carcinoma. Mol Cytogenet 8:78, 2015

27. Maemondo $M$, Inoue $A$, Kobayashi $K$, et al: Gefitinib or chemotherapy for non-small-cell lung cancer with mutated EGFR. N Engl J Med 362: 2380-2388, 2010 
28. Douillard JY, Oliner $K S$, Siena $S$, et al: Panitumumab-FOLFOX4 treatment and RAS mutations in colorectal cancer. N Engl J Med 369: 1023-1034, 2013

29. Cappuzzo F, Finocchiaro G, Grossi F, et al: Phase II study of afatinib, an irreversible ErbB family blocker, in EGFR FISH-positive non-small-cell lung cancer. J Thorac Oncol 10:665-672, 2015

30. Martin V, Mazzucchelli L, Frattini M: An overview of the epidermal growth factor receptor fluorescence in-situ hybridisation challenge in tumour pathology. J Clin Pathol 62:314-324, 2009

31. Kogawa T, Fouad TM, Liu DD, et al: High HER2/centromeric probe for chromosome 17 fluorescence in-situ hybridization ratio predicts pathologic complete response and survival outcome in patients receiving neoadjuvant systemic therapy with trastuzumab for HER2-overexpressing locally advanced breast cancer. Oncologist 21:21-27, 2016

32. Drenckhan A, Grob $T$, Dupree $A$, et al: Esophageal carcinoma cell line with high EGFR polysomy is responsive to gefitinib. Langenbecks Arch Surg 399:879-888, 2014

33. Huang J, Fan $Q$, Lu $P$, et al: Icotinib in patients with pretreated advanced esophageal squamous cell carcinoma with EGFR overexpression or EGFR gene amplification: A single-arm, multicenter phase 2 study. J Thorac Oncol 11:910-917, 2016

34. Bang YJ, Van Cutsem E, Feyereislova A, et al: Trastuzumab in combination with chemotherapy versus chemotherapy alone for treatment of HER2positive advanced gastric or gastro-oesophageal junction cancer (ToGA): A phase 3, open-label, randomised controlled trial. Lancet 376:687-697, 2010

35. Hecht JR, Bang YJ, Qin SK, et al: Lapatinib in combination with capecitabine plus oxaliplatin in human epidermal growth factor receptor 2-positive advanced or metastatic gastric, esophageal, or gastroesophageal adenocarcinoma: TRIO-013/LOGiC-a randomized phase III trial. J Clin Oncol 34:443-451, 2016
36. Secrier M, Li X, de Silva N, et al: Mutational signatures in esophageal adenocarcinoma define etiologically distinct subgroups with therapeutic relevance. Nat Genet 48:1131-1141, 2016

37. Kim J, Fox C, Peng S, et al: Preexisting oncogenic events impact trastuzumab sensitivity in ERBB2-amplified gastroesophageal adenocarcinoma. J Clin Invest 124:5145-5158, 2014

38. Pavlakis $N$, Sjoquist KM, Martin AJ, et al: Regorafenib for the treatment of advanced gastric cancer (INTEGRATE): A multinational placebo-controlled phase II trial. J Clin Oncol 34:2728-2735, 2016

39. Yang $Y L, X u K L, Z h o u ~ Y$, et al: Correlation of epidermal growth factor receptor overexpression with increased epidermal growth factor receptor gene copy number in esophageal squamous cell carcinomas. Chin Med J (Engl) 125:450-454, 2012

40. Jiang $D$, Li $X$, Wang $H$, et al: The prognostic value of EGFR overexpression and amplification in esophageal squamous cell carcinoma. BMC Cancer $15: 377,2015$

\section{Affiliations}

Russell D. Petty, University of Dundee; Asa Dahle-Smith, Ninewells Hospital and Medical School, Dundee; David A.J. Stevenson, Aileen Osborne, Doreen Massie, Caroline Clark, Zosia Miedzybrodzka, and Graeme I. Murray, University of Aberdeen, Aberdeen; Susan J. Dutton and Corran Roberts, Centre for Statistics in Medicine, University of Oxford, Oxford; Mark Harrison, Mount Vernon Hospital, Northwood; Irene Y. Chong and Ian Chau, Royal Marsden Hospital, London and Surrey; Wasat Mansoor, Christie Hospital, Manchester; Joyce Thompson, Birmingham Heartland Hospital, Heart of England National Health Service Trust, Birmingham; Anirban Chatterjee, Royal Shrewsbury Hospital, Shrewsbury; Stephen J. Falk, Bristol Oncology Centre, Bristol; Sean Elyan, Cheltenham General Hospital, Cheltenham; Angel Garcia-Alonso, Clan Clwyd Hospital, Rhyl; David Walter Fyfe, Furness General Hospital, Furness; Jonathan Wadsley, Weston Park Hospital, Sheffield, United Kingdom; and David R. Ferry, Eli Lilly and Company, Bridgewater, NJ.

\section{Support}

This work was funded by the Scottish Government, Chief Scientists Office (Grant No. ETM/116) and the Grampian Gastroesophageal Cancer Research Fund. I.C. and I.Y.C. were supported through the National Health Service funding to the National Institute for Health Research Biomedical Research Centre at the Royal Marsden NHS Foundation Trust and the Institute of Cancer Research.

\section{Prior Presentation}

Presented in part at the American Society of Clinical Oncology Annual Meeting, Chicago, IL, May 30-June 3, 2014.

\section{Explore the ASCO Cancer Genetics Program, a Comprehensive eLearning Program Focusing on Hereditary Cancer Genetics}

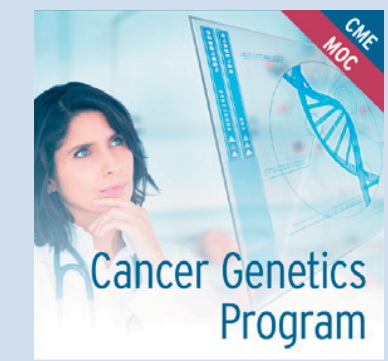

The ASCO Cancer Genetics Program, which includes 10 site-specific sections, allows you to gain greater competence in an array of topics related to the genetic cancer risk assessment process and addresses ways to improve the taking and documenting of family history as well as the interpreting of family history results. Earn CME/CE credits and ABIM MOC points. ASCO members save $20 \%$. Learn more about this course at university.asco.org. This course is part of ASCO University Essentials and Advanced Practitioner Certificate Programs. 
AUTHORS' DISCLOSURES OF POTENTIAL CONFLICTS OF INTEREST

Gefitinib and EGFR Gene Copy Number Aberrations in Esophageal Cancer

The following represents disclosure information provided by authors of this manuscript. All relationships are considered compensated. Relationships are self-held unless noted. I = Immediate Family Member, Inst = My Institution. Relationships may not relate to the subject matter of this manuscript. For more information about ASCO's conflict of interest policy, please refer to www.asco.org/rwc or ascopubs.org/jco/site/ifc.

Russell D. Petty

Honoraria: Eli Lilly, Pfizer

Consulting or Advisory Role: Eli Lilly, Bristol-Myers Squibb UK Speakers' Bureau: Pfizer

Research Funding: AstraZeneca, Merck KGaA, Eli Lilly, Boston

Biomedical, Janssen

Travel, Accommodations, Expenses: Eli Lilly, Merck KGaA, Bristol-Myers Squibb UK

Asa Dahle-Smith

Travel, Accommodations, Expenses: Merck, Astellas

David A.J. Stevenson

Employment: Roche (I)

Honoraria: Roche

Travel, Accommodations, Expenses: Roche

\section{Aileen Osborne}

No relationship to disclose

Doreen Massie

No relationship to disclose

Caroline Clark

No relationship to disclose

Graeme I. Murray

Research Funding: Vertebrate Antibodies (Inst)

Susan J. Dutton

No relationship to disclose

Corran Roberts

No relationship to disclose

Irene Y. Chong

Research Funding: Janssen Oncology

Wasat Mansoor

No relationship to disclose

Joyce Thompson

Honoraria: Pfizer

Consulting or Advisory Role: Roche, Chugai Pharmaceutical, Novartis Travel, Accommodations, Expenses: Novartis

\section{Mark Harrison}

No relationship to disclose

Anirban Chatterjee

Honoraria: Pfizer

Consulting or Advisory Role: Eli Lilly

Travel, Accommodations, Expenses: Roche, Pfizer

Stephen J. Falk

Research Funding: Cellgene (Inst), Gilead Sciences (Inst)

Travel, Accommodations, Expenses: Cellgene

Sean Elyan

No relationship to disclose

Angel Garcia-Alonso

No relationship to disclose

David Walter Fyfe

No relationship to disclose

Jonathan Wadsley

Honoraria: AstraZeneca, Genzyme, Eisai, Celgene, Bayer, Eli Lilly, Baxalta, Novartis

Consulting or Advisory Role: Eisai, Novartis

Research Funding: Astra Zeneca, Sanofi Genzyme

Travel, Accommodations, Expenses: Celgene, Novartis, SOBI, Ipsen

Ian Chau

Honoraria: Taiho Pharmaceutical, Pfizer, Eli Lilly, Amgen, Gilead Sciences Consulting or Advisory Role: Sanofi, Eli Lilly, Bristol-Myers Squibb, MSD Oncology, Bayer, Roche, Five Prime Therapeutics

Research Funding: Janssen-Cilag (Inst), Sanofi (Inst), Merck Serono (Inst) Travel, Accommodations, Expenses: MSD Oncology, Merck Serono, Sanofi, Eli Lilly

David R. Ferry

No relationship to disclose

Zosia Miedzybrodzka

Research Funding: Amgen

Travel, Accommodations, Expenses: Alexion Pharmaceuticals, MSD

Oncology, Mylan, Akcea, Sanofi (I), Amgen, AstraZeneca 


\section{Acknowledgment}

We thank the NHS Grampian Biorepository for their assistance with the management of biological specimens. The TRANS-COG study principal investigators are listed in the Data Supplement. 